\title{
Downregulation of miRNA-26b inhibits cancer proliferation of laryngeal carcinoma through autophagy by targeting ULK2 and inactivation of the PTEN/AKT pathway
}

\author{
SHUJING WANG ${ }^{1 *}$, DANDAN GUO ${ }^{1 *}$ and CONGYING LI $^{2}$ \\ ${ }^{1}$ Huaihe Hospital of Henan University; ${ }^{2}$ Medical College of Kaifeng University, \\ Gulou, Kaifeng, Henan 475000, P.R. China
}

Received November 12, 2016; Accepted June 7, 2017

DOI: $10.3892 /$ or.2017.5804

\begin{abstract}
Laryngeal carcinoma is one of the most common tumors of the head and neck cancers, the pathogenesis of which remains yet unclear. It has been discovered through research that microRNAs (miRNAs) play an important role during the genesis of laryngeal carcinoma. In the present study we investigated the effect of miRNA-26b on the proliferation of laryngeal carcinoma and elucidated the potential underlying mechanisms in order to provide new targets for laryngeal carcinoma. Firstly, we found that miRNA-26b expression was significantly increased in patients with laryngeal carcinoma, compared with normal volunteers. The downregulation of miRNA-26b inhibited cell proliferation and induced apoptosis of Hep-2 cells. Furthermore, downregulation of the expression of miRNA-26b promoted Bax, LC3 and p62 protein expression, decreased ULK2 mRNA and protein expression, as well as PTEN protein expression and increased phosphorylated-AKT protein expression in Hep-2 cells as determined using quantification by real-time PCR and western blotting. The concomitant downregulation of ULK2 and miRNA-26b futher enhanced the miRNA-26b-induced autophagy and apoptosis in addition to the miRNA-26b-inhibited cell proliferation of Hep-2 cells by targeting ULK2 and inactivating the PTEN/AKT pathway as determined by immunocytofluorescence. These findings revealed that miRNA-26b may play a key role in cell growth and death of laryngeal carcinoma through ULK2 and the PTEN/AKT pathway, and thus may be a new target for gene therapy in laryngeal carcinoma.
\end{abstract}

Correspondence to: Dr Shujing Wang, Huaihe Hospital of Henan University, 8 Baobei Road, Gulou, Kaifeng, Henan 475000, P.R. China

E-mail:wdfa3519619@126.com

${ }^{*}$ Contibuted equally

Key words: laryngeal carcinoma, miRNA-26b, autophagy, ULK2, PTEN/AKT

\section{Introduction}

Head and neck cancer is the sixth most commonly observed malignancy, and laryngocarcinoma is one of the most commonly observed malignancies among the head and neck tumors. Its morbidity ranks in the second place in respiratory tract neoplasms, accounting for $\sim 95 \%$ (1). With industrialized development and aggravated air pollution, the morbidity of laryngocarcinoma exhibits a gradual increasing trend in China as well as worldwide (2). As documented, the morbidity of laryngocarcinoma is increasing at a rate of $\sim 25 \%$ each year, and it is commonly observed in middle- and old-aged men (2). According to the latest research, though novel surgical treatments, chemotherapy drugs, more advanced radiotherapy means and targeted drugs have been applied in treating laryngocarcinoma in the last 30 years, the overall survival of patients with laryngocarcinoma has not improved, instead, it even shows a decreasing trend, which is $\sim 50 \%$; and that of advanced stage laryngocarcinoma is even as low as $30-40 \%$. The overall therapeutic effects are unsatisfying (3).

MicroRNA (miRNA), a type of non-coding RNA that consists of 22 nucleotides and was discovered in recent years, has an extensive gene expression regulation effect in vivo (4). miRNAs are closely correlated with the genesis and development of tumors, and they are attracting more and more attention in regards to the early diagnosis, metastasis monitoring, targeted therapy and prognosis of tumors (5). An miRNA regulates the biological characteristics of a tumor either by behaving as the tumor-suppressor gene which downregulates the activity of the proto-oncogene, or acting as the cancer-promoting gene to downregulate the activity of the tumor-suppressor gene (6). In numerous diseases, the expression profile of an miRNA exhibits certain characteristic alterations, which are particularly obvious in tumors, in which the expression profiles of the miRNA in the tumor tissue and the normal paracarcinomatous tissue show marked differences (5). It has been determined that analyzing the specific miRNA levels of various tumors contributes to the early diagnosis and prognosis evaluation of the tumor, and miRNAs show promise as novel molecular markers for the diagnosis, recurrence, metastasis and prognosis of multiple malignancies (7). Zhang et al reported that $\mathrm{miRNA}-26 \mathrm{a} / \mathrm{b}$ regulate DNA replication licensing, 
tumorigenesis, and prognosis of lung cancer (8). Zhang et al reported that miRNA-26b suppresses colon cancer cell proliferation via LEF1 (9).

With in-depth research on the chemoradiotherapyresistance mechanism of tumor cells under a hypoxic microenvironment, increasing importance has been bestowed to a type of novel protein degradation process, which is called autophagy. Autophagy is a type of lysosomal degradation process that is essential to cellular survival, differentiation and development as well as homeostasis. In addition, it recycles nutrients, guarantees the dynamic balance between the synthesis and degradation of the intracellular protein, regulates and defends against cell stress, and thus maintains the normal function of cells through the degradation of the intracellular macromolecular substances and aging organelles $(10,11)$.

The precise process of autophagy includes the beginning of the autophagosome formation, extension, closure, maturity and degradation, the molecular basis of which is the activation of the protein sequence encoded by the autophagy-associated gene (12). The ATG gene was first discovered in yeast cells, and ATG1 is a type of serine/threonine protein kinase, which is essential to the initial period of autophagy (13). ATG1 has five homologous proteins in mammals, which are Ulk1-4 and STK36 (13). The major functional molecules involved in the precise autophagy process can be roughly divided into 4 groups: the first one is the ATG1/unc-51-like kinase (Ulk) complex; apart from Ulk, the ATG13 and FIP200 scaffolds are also included (it is considered as the autoploid of yeast ATG17) (14).

PTEN is a type of tumor-suppressor gene, and tumorsuppressor genes are types of growth regulatory genes or negative regulatory genes, which can inhibit the transformation of normal cells into malignant cells under normal conditions (15). When a gene mutation, a gene deletion or gene inactivation develops and the gene loses its function, it may induce the malignant transformation of normal cells (7). The protein encoded by PTEN, which manifests the dual-specificity phosphatase activities of the lipid phosphatase activity and the protein phosphatase activity in the cytoplasm, is the first tumor-suppressor gene possessing phosphatase activity that has been discovered to date, and the lipid phosphatase activity is the main functional foundation for PTEN to inhibit a tumor (16). It plays a critical role in suppressing cell inhibition of apoptosis, cell cycle arrest, cell migration and inhibiting angiogenesis (17).

AKT is a type of protein kinase that plays an important role in biological behaviors such as cell proliferation, differentiation, apoptosis and migration, and it can phosphorylate serine/threonine (18). The AKT family members are comprised of three subtypes, namely, AKT1, AKT2 and AKT3, and they are also named $\mathrm{PKB} \alpha, \mathrm{PKB} \beta$ and $\mathrm{PKB} \gamma$ (18). P-AKT influences the activation of multiple downstream effector molecules through the phosphorylation cascade reaction and the interaction with the target protein, thus regulating multiple biological effects, such as cell growth and proliferation, regulation of angiogenesis, cell apoptosis and motion control (19). Current research suggests that its out-of-control expression is closely related to multiple tumors, and P-AKT is abundantly expressed in tumor tissues (20). To gain a better understanding of the association between miRNA-26b expression and laryngeal cancer, we studied the effect of miRNA-26b on the proliferation of laryngeal carcinoma and elucidated the potential underlying mechanisms to provide new targets for laryngeal carcinoma.

\section{Materials and methods}

Human tissue samples. Laryngeal carcinoma patients $(\mathrm{n}=59)$ and healthy volunteers $(n=8)$ and were obtained from the Huaihe Hospital of Henan University. Whole blood was obtained and centrifuged at 2,000 x g for $10 \mathrm{~min}$. Serum was obtained and saved at $-80^{\circ} \mathrm{C}$. Every two months, we surveyed palindromia or survival rate of all laryngeal carcinoma patients.

Quantification by real-time PCR. Total RNA was isolated from serum using RNAiso Plus Reagent according to the manufacturer's instructions. cDNA was generated following the SuperScript II RT Protocol (Invitrogen, Carlsbad, CA, USA). qRT-PCR was performed using an Applied Biosystems 7500 Fast Real-Time PCR System with the SYBR-Green PCR Master Mix protocol (Applied Biosystems, Foster City, $\mathrm{CA}$, USA). The reactions were incubated at $95^{\circ} \mathrm{C}$ for $5 \mathrm{~min}$, followed by 40 cycles of $95^{\circ} \mathrm{C}$ for $20 \mathrm{sec}, 60^{\circ} \mathrm{C}$ for $30 \mathrm{sec}$, and $72^{\circ} \mathrm{C}$ for $30 \mathrm{sec}$.

Cell lines and transfection. Squamous cell carcinoma of the larynx cell line Hep-2 were cultured in RPMI-1640 medium, $10 \%$ fetal bovine serum (FBS; Invitrogen, Shanghai, China) and penicillin/streptomycin at $37^{\circ} \mathrm{C}$ and $5 \% \mathrm{CO}_{2}$. The Hep-2 cells were then transfected with $100 \mathrm{nM}$ anti-miR-26 and si-ULK2 or negative control mimics (RiboBio, Guangzhou, China) using Lipofectamine 3000 (Invitrogen, Guangzhou, China).

Cell proliferation assay. After transfection at 24,48 and $72 \mathrm{~h}$, the cells were cultivated into 96 -well plates and incubated with MTT solution $(0.5 \mathrm{mg} / \mathrm{ml})$ for $4 \mathrm{~h}$ at $37^{\circ} \mathrm{C}$. The reaction was stopped by lysing the cells with $200 \mu \mathrm{l}$ of dimethyl sulfoxide (DMSO) for $5 \mathrm{~min}$. The absorbance was recorded at $490 \mathrm{~nm}$.

Apoptosis assay. Following transfection at $48 \mathrm{~h}$, the cells were cultivated into 6-well plates, and stained with an Annexin V-FITC/propidium iodide (PI) staining kit (BD Biosciences, San Jose, CA, USA) for $15 \mathrm{~min}$ at $4^{\circ} \mathrm{C}$. Analysis was performed using a FACScan flow cytometer (Becton-Dickinson, Bedford, MA, USA).

Caspase-3 and -9 assay. After transfection at $48 \mathrm{~h}$, the cells were collected and lysed with lysis buffer (CoWin Biotechnology, Beijing, China) on ice for $30 \mathrm{~min}$. Lysates were cleared by centrifugation at $10,000 \mathrm{x}$ f for $15 \mathrm{~min}$ at $4^{\circ} \mathrm{C}$. The protein concentrations were determined using BCA protein assay kits (CoWin Biotechnology). Proteins samples $(10 \mu \mathrm{g})$ were incubated with caspase- 3 and -9 assay kits for $1 \mathrm{~h}$ at $37^{\circ} \mathrm{C}$. The absorbance was recorded at $405 \mathrm{~nm}$.

Western blot analysis. After transfection at $48 \mathrm{~h}$, the cells were collected and lysed with lysis buffer (CoWin Biotechnology) on ice for $30 \mathrm{~min}$. Lysates were cleared by centrifugation at $10,000 \mathrm{xg}$ for $15 \mathrm{~min}$ at $4^{\circ} \mathrm{C}$. The protein concentrations were determined using $\mathrm{BCA}$ protein assay 
kits (CoWin Biotechnology). Proteins samples (50 $\mu \mathrm{g})$ were separated by $6-10 \%$ sodium dodecylsulfate-polyacrylamide gel electrophoresis (SDS-PAGE), and then transferred onto polyvinylidine fluoride (PVDF) membranes (Thermo Fisher Scientific, Inc., Waltham, MA, USA). The membranes were incubated with Bax, LC3, p62, PTEN, phosphorylated (p)-AKT, ULK2 (1:2,000; all from Cell Signaling, Shanghai, China) and GAPDH $\left(1: 1,000\right.$; Beyotime, Shanghai, China) overnight at $4^{\circ} \mathrm{C}$. The membranes were then washed with Tris-buffered saline and Tween-20 (TBST), and then incubated with a horseradish peroxidase-conjugated secondary antibody (1;5000; Cell Signaling) for $1 \mathrm{~h}$ at room temperature (RT). The protein expression was detected with an electrochemiluminescence (ECL) detection reagent (Beyotime) and quantified using Quantity One software (Bio-Rad, Hercules, CA, USA).

Immunocytofluorescence. After transfection at $48 \mathrm{~h}$, the cells were seeded onto cell chamber slides and fixed with $4 \%$ formaldehyde at $4^{\circ} \mathrm{C}$ for $15 \mathrm{~min}$. The cells were then stained with the ULK2 antibody (1:500; Cell Signaling) at $4^{\circ} \mathrm{C}$ overnight. Subsequently, the cells were incubated with a secondary antibody (donkey anti-rabbit FITC; 1:100 dilution; Santa Cruz Biotechnology, Santa Cruz, CA, USA) for $1 \mathrm{~h}$ at RT in the dark. Nuclei were counterstained with 4',6-diamidino-2-phenylindole (DAPI) at RT for $15 \mathrm{~min}$, and images were captured using an automated upright microscope system (Leica DM4000B; Leica Microsystems, Wetzlar, Germany).

Statistical analysis. Results are expressed as the mean \pm SD. One-way analysis of variance (ANOVA) was used to analyze the significance between groups. A P-value of $<0.05$ was considered to indicate a statistically significant difference.

\section{Results}

miRNA-26b expression in patients with laryngeal carcinoma. Firstly, we analyzed miRNA-26b expression in patients with laryngeal carcinoma and normal volunteers. We found that miRNA-26b expression was significantly increased in patients with laryngeal carcinoma, compared with normal volunteers (Fig. 1).

Relationship between miRNA-26b expression and clinical TNM stage of patients with laryngeal carcinoma. We analyzed the relationship between miRNA-26b expression and the clinical tumor-node-metastasis (TNM) stage of patients with laryngeal carcinoma to explore the effects of miRNA-26b on laryngeal carcinoma. As shown in Fig. 2, the expression of miRNA-26b in stages I-II, III or IV of patients with laryngeal carcinoma was significantly increased compared with normal volunteers, and the miRNA-26b expression of stage IV patients with laryngeal carcinoma was higher than that of stage I-II patients with laryngeal carcinoma. These results revealed that the expression of miRNA-26b was increased in patients with laryngeal carcinoma as well as with its progression.

Relationship between the expression of miRNA-26b and the survival rate of patients with laryngeal carcinoma. Next, we also studied the relationship between the expression of miRNA-26b and the survival rate of patients with laryngeal

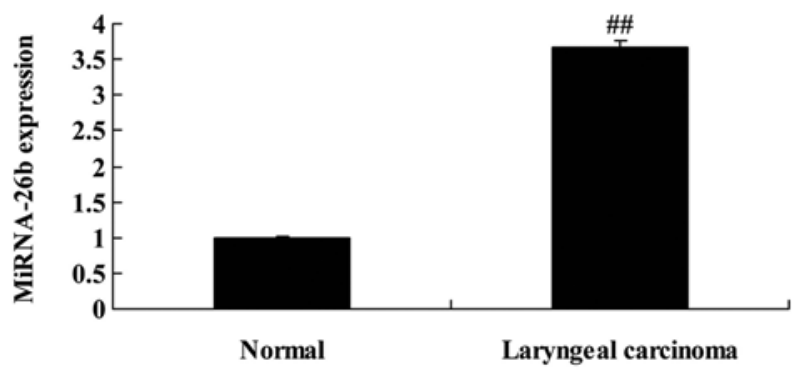

Figure 1. miRNA-26b expression in patients with laryngeal carcinoma.

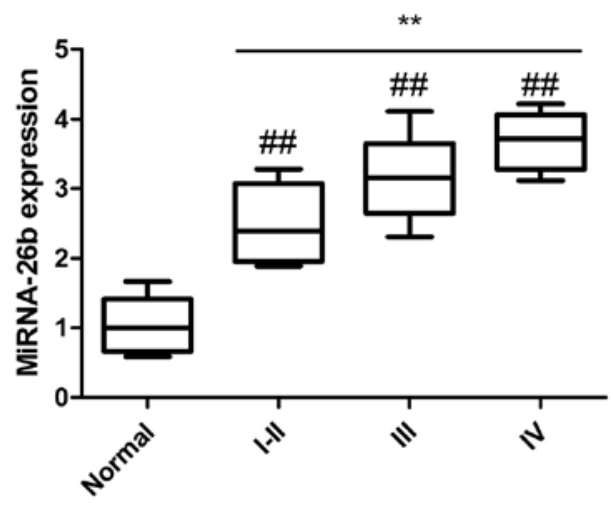

Figure 2. Relationship between the expression of miRNA-26b and the clinical TNM stage of patients with laryngeal carcinoma; ${ }^{* *} \mathrm{p}<0.01$ vs. the I-II group, ${ }^{\# \#} \mathrm{p}<0.01$ vs. the control group.

carcinoma. The overall survival (OS) and disease-free survival (DFS) of patients with laryngeal carcinoma with high expression of miRNA-26b were lower than those of patients with low expression of miRNA-26b (Fig. 3).

Downregulation of miRNA-26b inhibits cell proliferation and induces apoptosis of laryngeal carcinoma cells. The MTT assay and flow cytometric analysis with PI staining revealed that downregulation of miRNA-26b inhibited cell proliferation and induced apoptosis of laryngeal carcinoma cells, compared with the negative control group (Fig. 4).

Downregulation of miRNA-26b promotes caspase-3/-9 activities of laryngeal carcinoma cells. We examined whether the expression of miRNA-26b affects caspase-3/-9 activities of laryngeal carcinoma cells. Downregulation of miRNA-26b significantly promoted caspase-3/-9 activities of laryngeal carcinoma cells compared with the negative control group (Fig. 5).

Downregulation of miRNA-26b promotes Bax, LC3 and p62 protein expression of laryngeal carcinoma cells. We next assessed the functional role of miRNA-26b on autophagy of laryngeal carcinoma cells. Fig. 6 revealed that the downregulation of miRNA-26b significantly promoted Bax, LC3 and p62 protein expression of laryngeal carcinoma cells compared with the negative control group.

Downregulation of miRNA-26b decreases ULK2 $m R N A$ and protein expression as well as PTEN protein expression and 

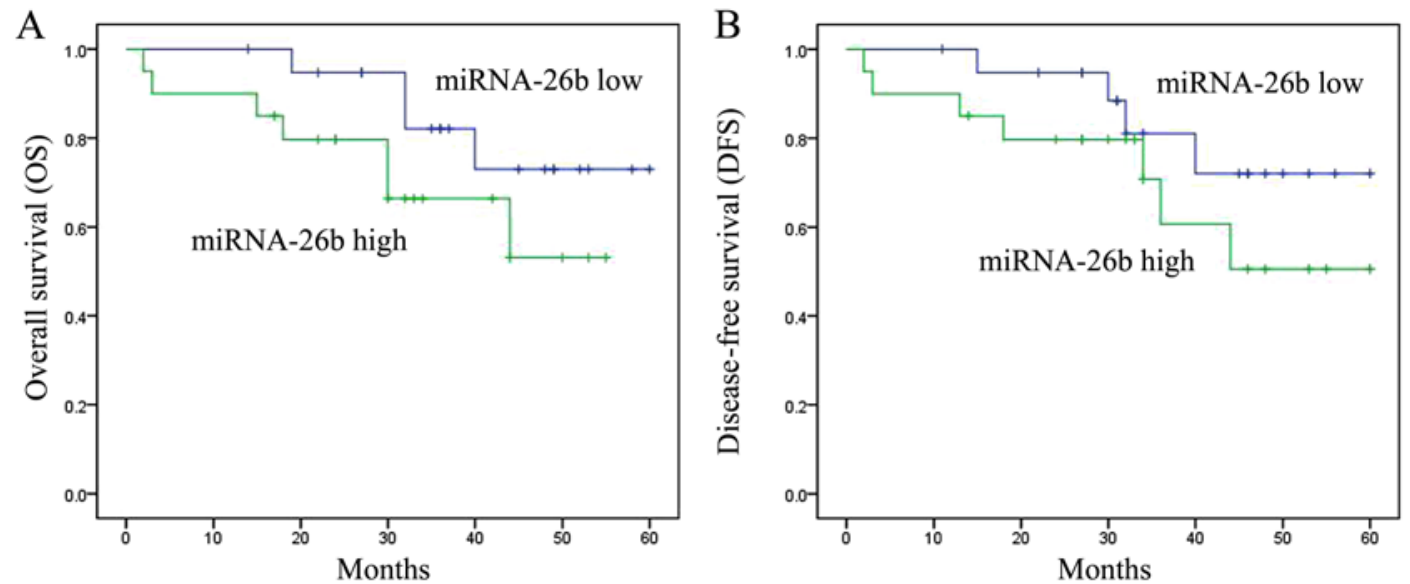

Figure 3. Relationship between the expression of miRNA-26b and the survival rate of patients with laryngeal carcinoma. Relationship between the expression of miRNA-26b and the (A) OS and (B) DFS of patients with laryngeal carcinoma; * ${ }^{*}<0.01$ vs. the control group.

A

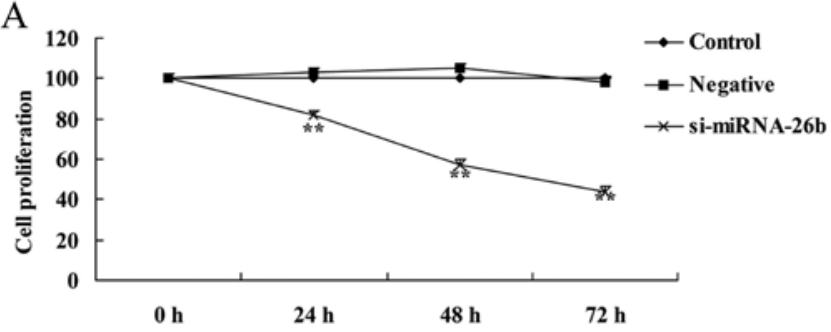

B

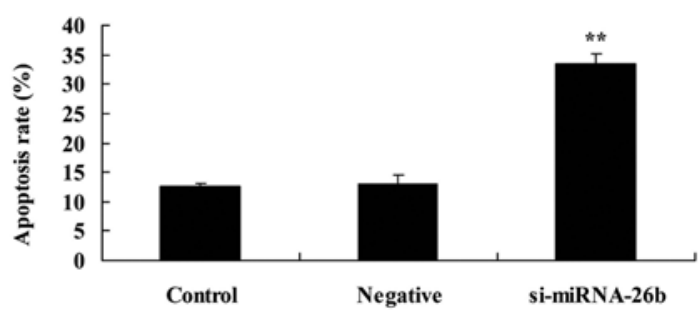

Figure 4. Downregulation of miRNA-26b inhibits cell proliferation and induced apoptosis of laryngeal carcinoma cells. Downregulation of miRNA-26b inhibited the (A) cell proliferation and (B) induced apoptosis of laryngeal carcinoma cells; * ${ }^{*}<<0.01$ vs. the control group.

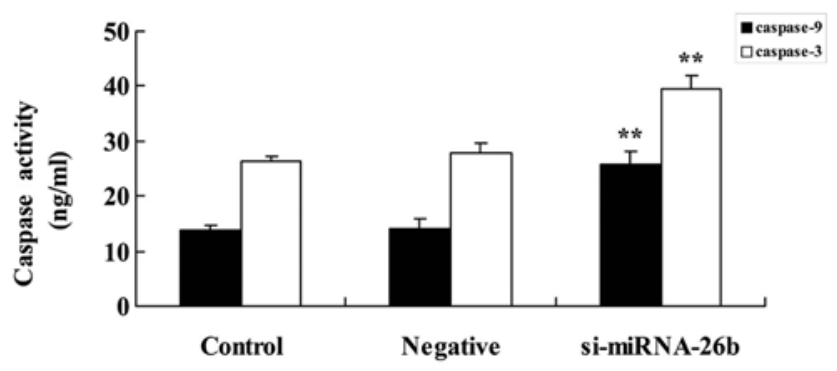

Figure 5. Downregulation of miRNA-26b promotes caspase- $3 /-9$ activities of laryngeal carcinoma cells. ${ }^{* *} \mathrm{p}<0.01$ vs. the control group.

increases $p$-AKT protein expression of laryngeal carcinoma cells. We further confirmed whether miRNA-26b affected ULK2 mRNA and protein expression as well as PTEN and p-AKT protein expression of laryngeal carcinoma cells. Fig. 7 revealed that the dowregulation of miRNA-26b decreased ULK2 mRNA and protein expression as well as PTEN protein expression and increased p-AKT protein expression of laryngeal carcinoma cells, compared with the negative control group.

Downregulation of miRNA-26b affects ULK2 protein expression of laryngeal carcinoma cells as determined using immunocytofluorescence. We used immunocytofluorescence to observe ULK2 protein expression of laryngeal carcinoma cells. Fig. 8 revealed that the downregulation of miRNA-26b promoted inhibition of ULK2 protein expression of laryngeal carcinoma cells.
Concomitant downregulation of ULK2 and miRNA-26b enhances the miRNA-26b-inhibited PTEN and miRNA-26b-induced -AKT protein expression of laryngeal carcinoma cells. To address the potential involvement of ULK2 in the effects of miRNA-26b on laryngeal carcinoma cells, si-ULK2 was used to inhibit laryngeal carcinoma cells. As shown in Fig. 9, the concomitant downregulation of ULK2 and miRNA-26b significantly enhanced the miRNA-26b-inhibited ULK2 protein expression and mRNA expression, as well as the PTEN protein expression and the miRNA-induced p-AKT protein expression of laryngeal carcinoma cells, compared with the si-miRNA-26b group alone.

Concomitant downregulation of ULK2 and miRNA-26b enhances the miRNA-26b-inhibited cell proliferation and the miRNA-26b-induced apoptosis of laryngeal carcinoma cells. 


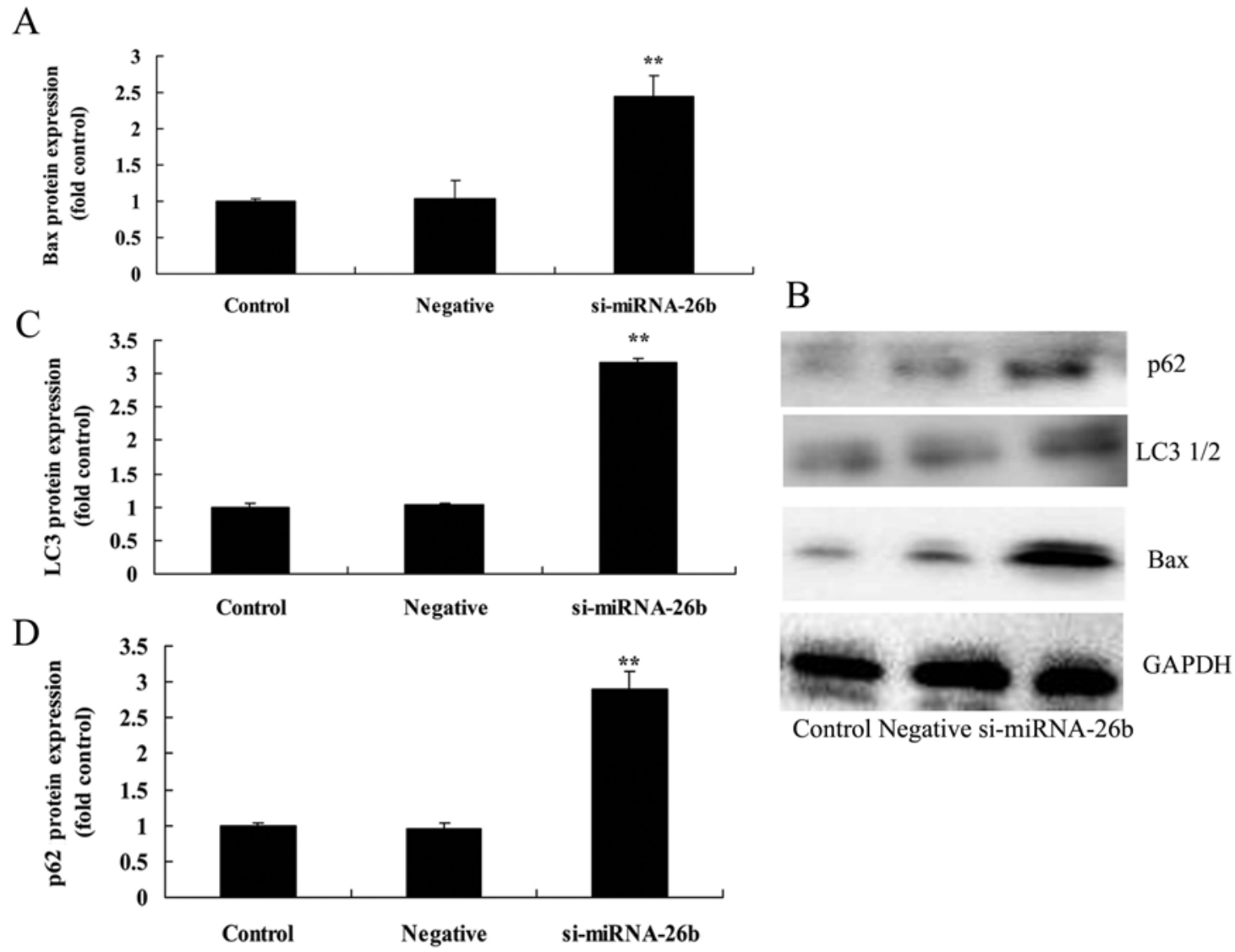

Figure 6. Downregulation of miRNA-26b promotes Bax, LC3 and p62 protein expression of laryngeal carcinoma cells. Downregulation of miRNA-26b promoted (A) Bax, (C) LC3 and (D) p62 protein expression as determined by statistical analysis and (B) western blot analysis of laryngeal carcinoma cells; ** $<<0.01$ vs. the control group.

A

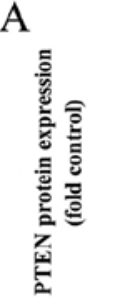

C

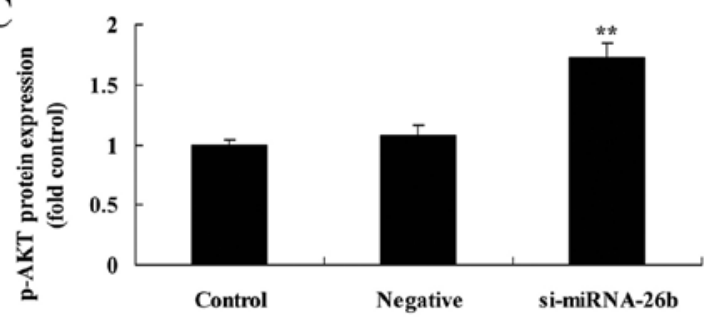

$\mathrm{D}$

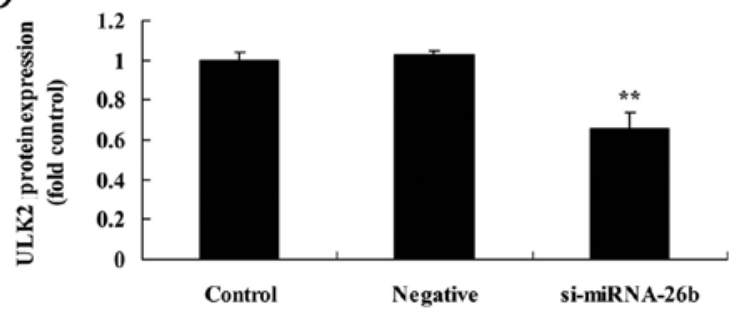

$\mathrm{B}$
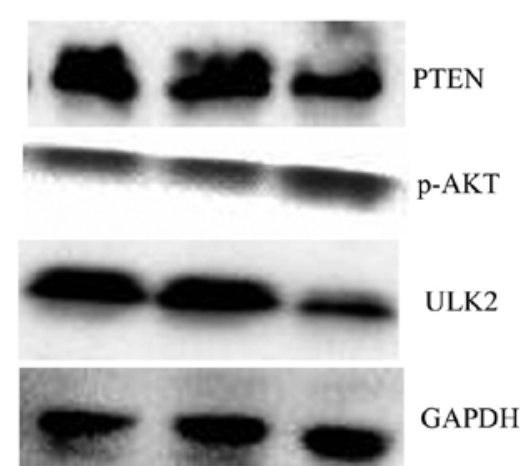

Control Negative si-miRNA-26b
$\mathrm{E}$

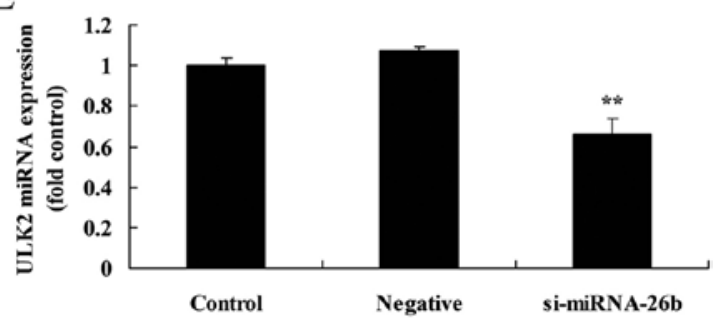

Figure 7. Downregulation of miRNA-26b decreases ULK2 mRNA and protein expression as well as PTEN protein expression and increases p-AKT protein expression of laryngeal carcinoma cells. (A, C and D) PTEN, p-AKT and ULK2 protein expression as determined by statistical analysis and (B) western blot analysis and (E) ULK2 mRNA expression; ${ }^{* *} \mathrm{p}<0.01$ vs. the control group. 


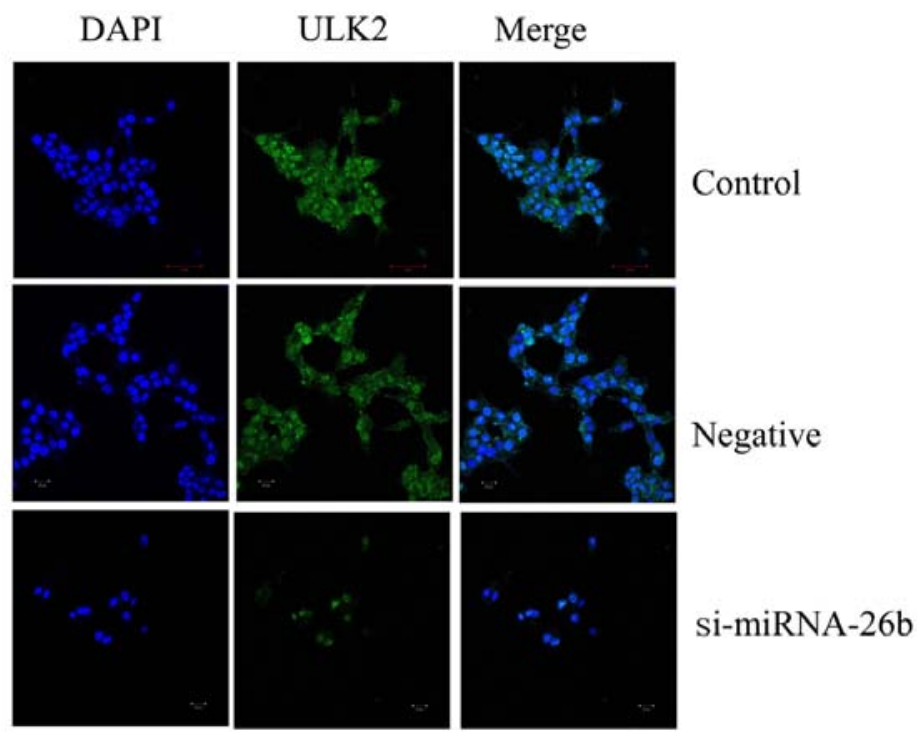

Figure 8. Downregulation of miRNA-26b affects ULK2 protein expression of laryngeal carcinoma cells as determined using immunocytofluorescence.

A

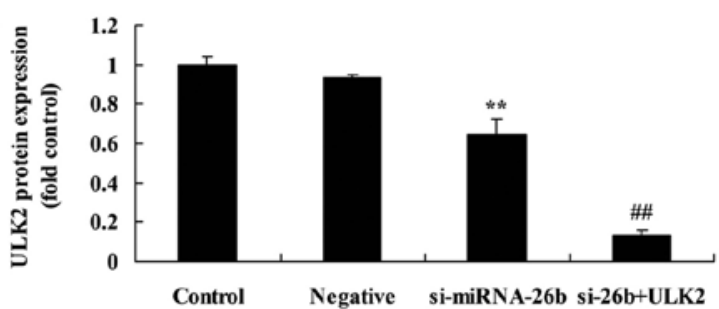

$\mathrm{C}$

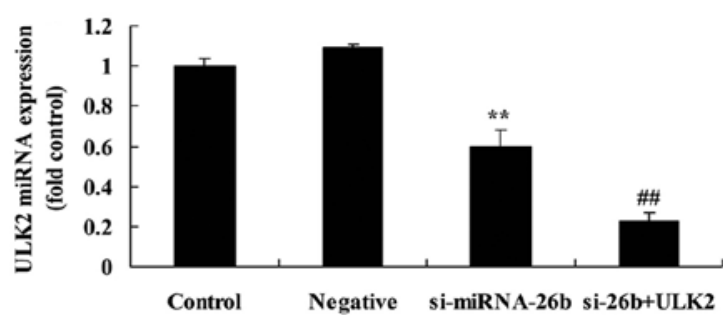

$\mathrm{D}$

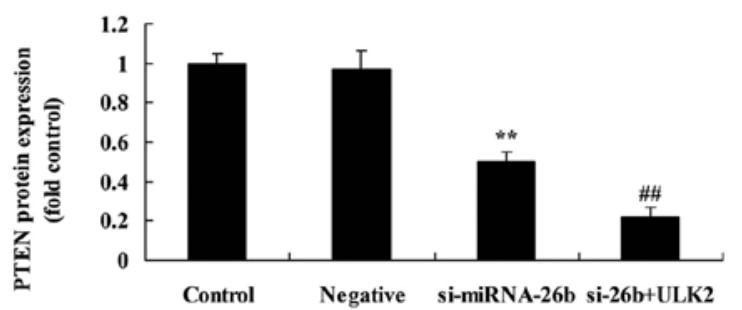

B
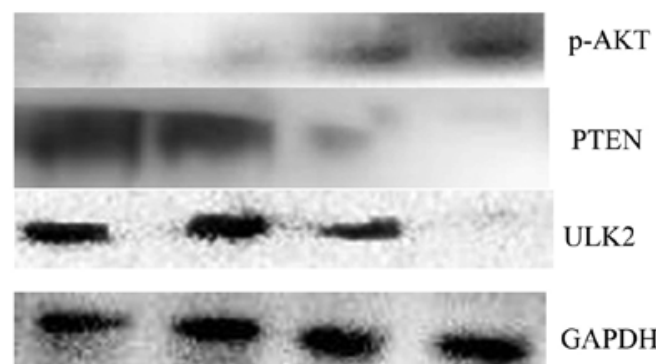

Control Negative si-miRNA-26b si-26b+ULK2
$\mathrm{E}$

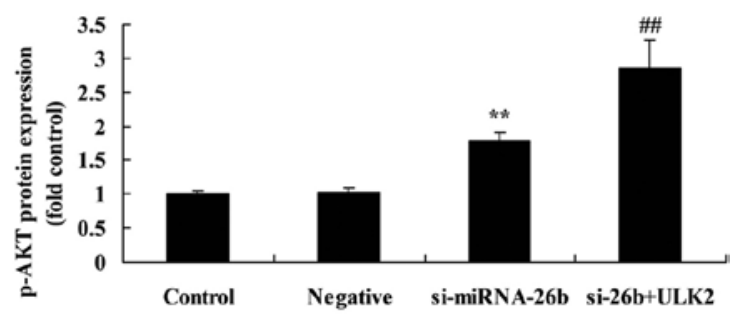

Figure 9. Concomitant downregulation of ULK2 and miRNA-26b enhances the miRNA-26b-inhibited ULK2 protein and miRNA expression as well as the PTEN protein expression and the miRNA-26b-induced p-AKT protein expression of laryngeal carcinoma cells. (A, D and E) ULK2, PTEN, p-AKT protein expression as determined by statistical analysis and (B) western blot analysis and (C) ULK2 mRNA expression; ${ }^{* *}$ p $<0.01$ vs. the control group, ${ }^{\# \#}$ p $<0.01$ vs. the control group.

In order to investigate the potential involvement of ULK2 in the effects of miRNA-26b on laryngeal carcinoma cells, the cell proliferation and induced apoptosis of laryngeal carcinoma cells were assessed. As shown in Fig. 10, the concomitant downregulation of ULK2 and miRNA-26b significantly enhanced the miRNA-26b-inhibited cell proliferation and the miRNA-26b-induced apoptosis of laryngeal carcinoma cells, compared with the si-miRNA-26b group alone.
Concomitant downregulation of ULK2 and miRNA-26b enhances the miRNA-26b-induced caspase-3/-9 activities of laryngeal carcinoma cells. We next investigated the caspase-3/-9 activities of laryngeal carcinoma cells after ULK2 downregulation. As shown in Fig. 11, the concomitant downregulation of ULK2 and miRNA-26b significantly increased the miRNA-26b-induced caspase-3/-9 activities of laryngeal carcinoma cells compared with the si-miRNA-26b group alone. 

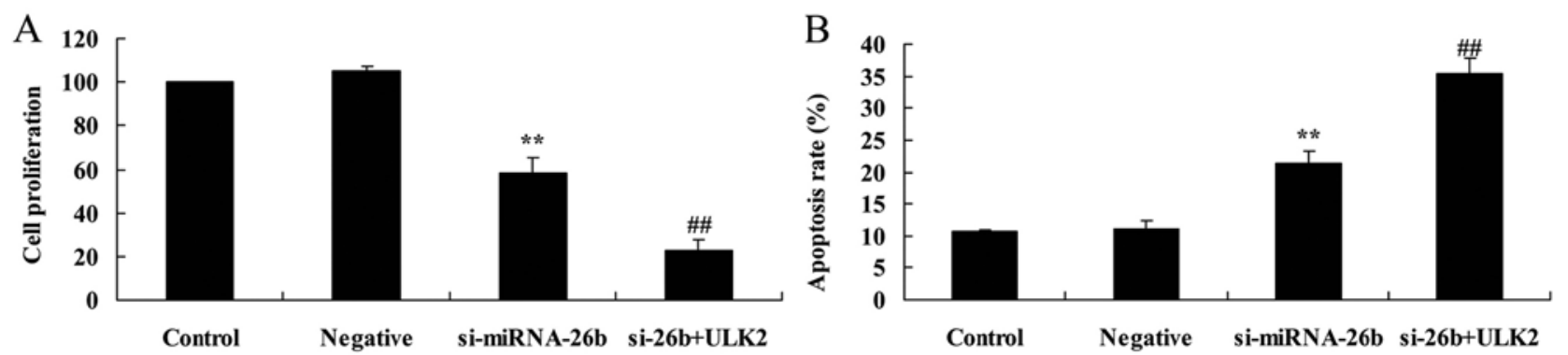

Figure 10. Concomitant downregulation of ULK2 and miRNA-26b enhances the miRNA-26b-inhibited cell proliferation and the miRNA-26b-induced apoptosis of laryngeal carcinoma cells. Concomitant downregulation of ULK2 and miRNA-26b enhanced (A) the miRNA-26b-inhibited cell proliferation and (B) the miRNA-26b-induced apoptosis of laryngeal carcinoma cells; ${ }^{* *} \mathrm{p}<0.01$ vs. the control group, ${ }^{\# \#} \mathrm{p}<0.01$ vs. the control group.

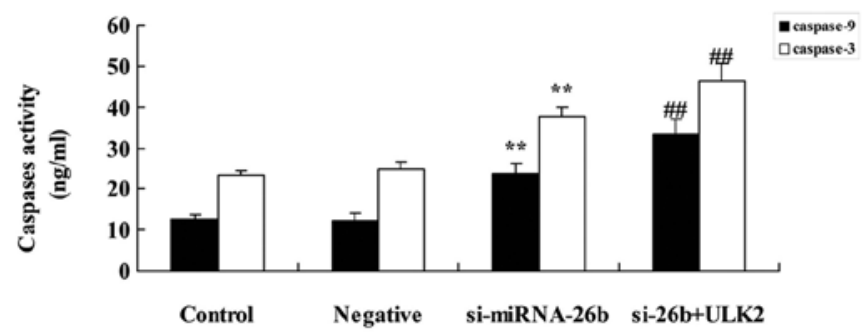

Figure 11. Concomitant downregulation of ULK2 and miRNA-26b enhances the miRNA-26b-induced caspase-3/-9 activities of laryngeal carcinoma cells; ${ }^{* * *} \mathrm{p}<0.01$ vs. the control group, ${ }^{\# \#} \mathrm{p}<0.01$ vs. the control group.

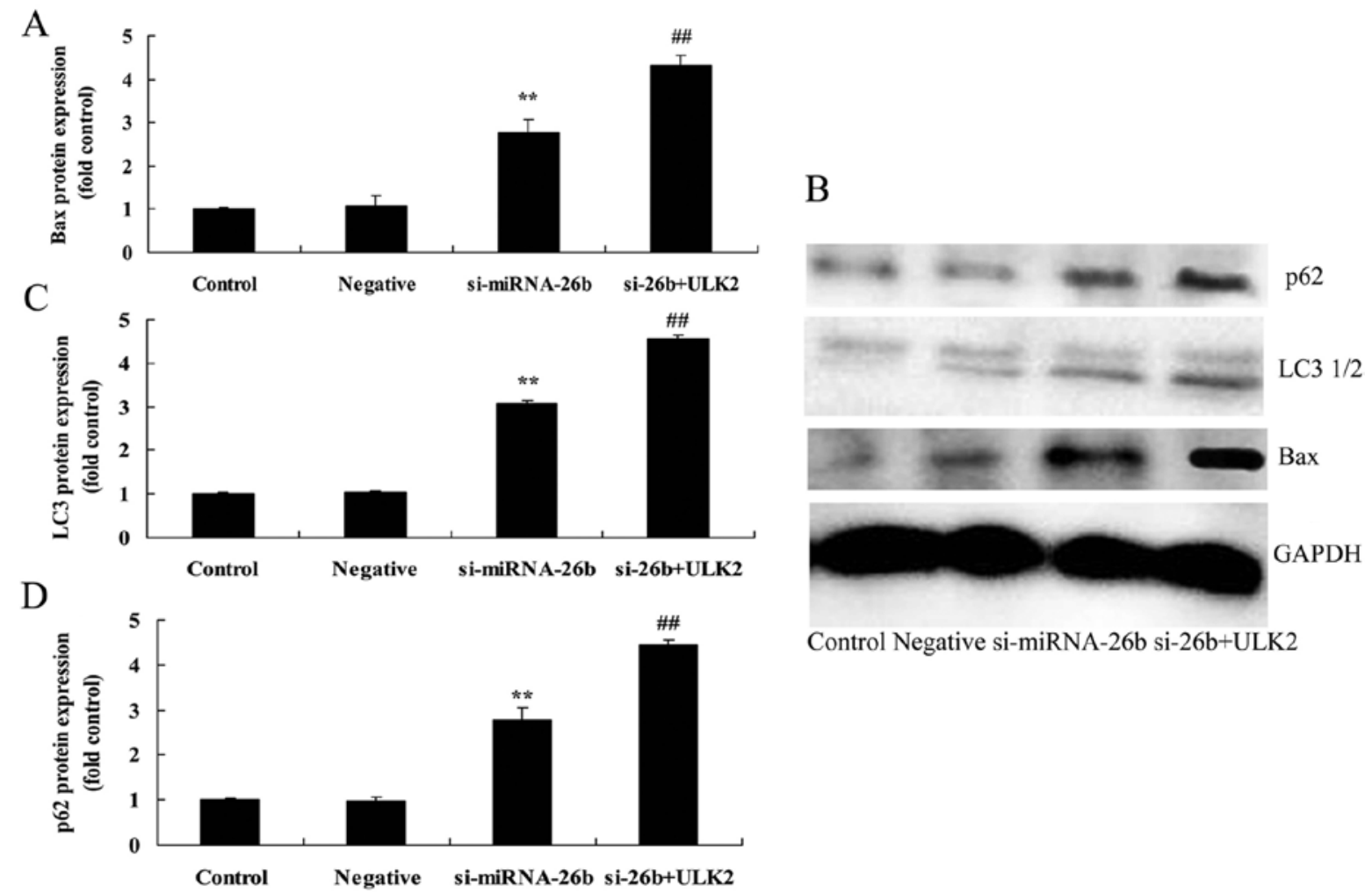

Figure 12. Concomitant downregulation of ULK2 and miRNA-26b enhances the miRNA-26b-induced Bax, LC3 and p62 protein expression of laryngeal carcinoma cells. Concomitant downregulation of ULK2 and miRNA-26b enhanced the miRNA-26b-induced (A) Bax, (C) LC3 and (D) p62 protein expression as determined by statistical and (B) western blot analyses of laryngeal carcinoma cells; ${ }^{* *} \mathrm{p}<0.01 \mathrm{vs}$. the control group, ${ }^{\# \#} \mathrm{p}<0.01 \mathrm{vs}$. the control group.

Concomitant downregulation of ULK2 enhances miRNA-26b-induced autophagy, Bax, LC3 and p62 protein expression of laryngeal carcinoma cells. In order to investigate the potential involvement of ULK2 in the miRNA-26b-induced autophagy of laryngeal carcinoma cells, Bax, LC3 and p62 protein expression of laryngeal carcinoma cells were assessed. 
As shown in Fig. 12, the concomitant downregulation of ULK2 and miRNA-26b significantly enhanced miRNA-26b-induced autophagy, Bax, LC3 and p62 protein expression of laryngeal carcinoma cells compared with the si-miRNA-26b group alone.

\section{Discussion}

Laryngocarcinoma is a type of malignancy with high morbidity, which accounts for $\sim 2.4 \%$ of newly-diagnosed malignancies worldwide each year (21). With the increasing development of the industrialization in China, the morbidity rate exhibits a gradual increasing trend. Approximately 2-4/100,000 cases of laryngocarcinoma belong to the second most commonly observed head and neck tumor, which appears mostly in middle- and old-aged men (22). It is generally considered that the genesis of laryngocarcinoma may be related to bad habits such as smoking, air pollution and occupational factors (22). Research in recent years revealed that a majority of tumor patients have improved survival. However, the survival rate of laryngocarcinoma has gradually decreased from 57.1 to $51.9 \%$ (23). Determining the survival of laryngocarcinoma at present may contribute to further assessing its trend. We found that miRNA-26b expression was significantly increased in patients with laryngeal carcinoma compared with normal volunteers.

Research on miRNA has provided a promising research direction for exploring the invasion and metastasis mechanism of laryngocarcinoma, searching for novel diagnostic markers and treatment targets (24). The in-depth research on the regulatory mechanism of miRNA is of great theoretical and practical significance in order to eventually reveal the mechanism of cell proliferation, differentiation and carcinogenesis of laryngocarcinoma, search for new diagnostic markers and treatment targets, and realize the effective and individualized treatment of tumors (25). In the present study we found that downregulation of miRNA-26b inhibited cell proliferation and induced apoptosis of Hep-2 cells.

It was discovered through in-depth research that autophagy cannot only inhibit the genesis of tumors, but can also promote the genesis of tumors (10). The various roles that autophagy plays in tumors may be related to the developmental process of a tumor. In the early stage of carcinogenesis, the inhibition of autophagy promotes the sustainable development of the primary cancer cell, thus exerting a distinct antitumor effect $(10,26)$. When the tumor lies in the growth and proliferative stages, the body improves the excessive growth of tumor cell-induced malnutrition and hypoxia through upregulation of autophagy and inhibition of the excessive proliferation of the tumor cells, and in this case, autophagy plays a tumor-promoting role (26). These results indicate that downregulation of miRNA-26b promoted LC3 and p62 protein expression in Hep-2 cells. Yuan et al revealed that HIF-2 $\alpha$-MALAT1-miR-216b regulates autophagy in hepatocellular carcinoma cells (27).

The beginning of autophagy is controlled by the Ulk $1 / 2$ kinase complex. They sense the signals from mTOR1, and when the autophagy begins, the aforementioned PI3K and Beclinl may form a complex, and the Ptlns3p it produces is necessary for the recruitment of other ATG proteins that are closely related to autophagy formation (28). Next, the formation of the ATG5-ATG12 complex plays an important role in the extension of the tunica media during the autophagosome formation process (29). This complex may recruit the cytosol-associated LC3, and transform it into LC3II which can combine with the autophagosome (30). LC3II binds with the membrane of the autophagosome under the action of ATG-3, and inserts itself between the inner and outer membrane of the autophagosome (30). After the autophagosome encapsulates the substances to be degraded, all types of ATG proteins return to the cell. Subsequently, the autophagosome binds with the lysosome and degrades the encapsulated substances (13). Furthermore, in the present study we found that downregulation of miRNA-26b decreased ULK2 mRNA and protein expression in Hep- 2 cells. Clotaire et al indicated that miRNA-26b inhibits autophagy through targeting ULK2 in prostate cancer cells (29).

The normal expression of the PTEN gene can inhibit the malignant transformation of the cell, and the mutation, deletion or inactivation of the gene may induce decreased expression of the protein, thus losing the antitumor effect, which frequently manifests as a mutation, a deletion or inactivation in multiple tumors (31). PTEN dephosphorylates the $\mathrm{PI}(3,4,5) \mathrm{P} 3$ in the $3^{\prime}$ carboxyl locus and forms $\mathrm{PI}(4,5) \mathrm{P} 2$, antagonizes the activity of PI3K, and promotes the progressive growth of a tumor $(16,32)$. These studies demonstrated that downregulation of miRNA-26b inhibited PTEN and induced p-AKT protein expression in Hep-2 cells in vitro, suggesting that miRNA-26b could be the target in cancer treatment via the PTEN/AKT signaling pathway. Palumbo et al suggested that the suppression of miRNA-26b regulates pituitary tumors through the regulation of the PTEN/AKT pathway (33).

The PI3K/Akt pathway is an important growth factor pathway in vivo, which can activate the anti-apoptotic mechanism, promote glucose metabolism and protein synthesis, thus promoting cell growth and proliferation (19). Abnormity in the signal transduction pathway may result in the abnormally increased cell growth, proliferation, metabolism and anti-apoptotic effects, which participate in the genesis and development of most tumors (34). Therefore, the PI3K/Akt pathway also named the anti-apoptotic pathway is considered as the primary pathway for cancer cell survival. Furthermore, we found that concomitant downregulation of ULK2 and miRNA-26 further enhanced miRNA-26b-induced inactivation of the PTEN/AKT pathway.

Collectively, we demonstrated that miRNA-26b regulates ULK2 and the PTEN/AKT pathway in laryngeal carcinoma autophagy and apoptosis. Based on our findings and those from others, miRNA-26b may play a key role in cell growth and death of laryngeal carcinoma and thus may be a new target for gene therapy in laryngeal carcinoma.

\section{References}

1. Miszczyk L, Maciejewski B, Tukiendorf A, Woźniak G, Jochymek B, Gawryszuk A and Szweda M: Split-course accelerated hyperfractionated irradiation (CHA-CHA) as a sole treatment for advanced head and neck cancer patients-final results of a randomized clinical trial. Br J Radiol 87: 20140212 , 2014.

2. Ghai B, Jain K, Bansal D and Bhatia N: End-tidal sevoflurane concentration for ProSeal ${ }^{\mathrm{TM}}$ versus Classic ${ }^{\mathrm{TM}}$ laryngeal mask airway insertion in unpremedicated anaesthetised adult females. Anaesth Intensive Care 44: 221-226, 2016. 
3. Takácsi-Nagy Z, Hitre E, Remenár É, Oberna F, Polgár C, Major T, Gödény M, Fodor J and Kásler M: Docetaxel, cisplatin and 5-fluorouracil induction chemotherapy followed by chemoradiotherapy or chemoradiotherapy alone in stage III-IV unresectable head and neck cancer: Results of a randomized phase II study. Strahlenther Onkol 191: 635-641, 2015.

4. Janoray G, Pointreau Y, Garaud P, Chapet S, Alfonsi M, Sire C, Jadaud $E$ and Calais G: Long-term results of a multicenter randomized phase III trial of induction chemotherapy with cisplatin, 5-fluorouracil, \pm docetaxel for larynx preservation. J Natl Cancer Inst 108: pii: djv368, 2015.

5. $\mathrm{Yu} \mathrm{X}$ and $\mathrm{Li} \mathrm{Z}$ : The role of microRNAs expression in laryngeal cancer. Oncotarget 6: 23297-23305, 2015.

6. Huang CX, Zhu Y, Duan GL, Yao JF, Li ZY, Li D and Wang QQ: Screening for miRNAs related to laryngeal squamous carcinoma stem cell radiation. Asian Pac J Cancer Prev 14: 4533-4537, 2013

7. Li JZ, Gao W, Lei WB, Zhao J, Chan JY, Wei WI, Ho WK and Wong TS: MicroRNA 744-3p promotes MMP-9-mediated metastasis by simultaneously suppressing PDCD4 and PTEN in laryngeal squamous cell carcinoma. Oncotarget 7: 58218-58233, 2016.

8. Zhang X, Xiao D, Wang Z, Zou Y, Huang L, Lin W, Deng Q, Pan H, Zhou J, Liang C, et al: MicroRNA-26a/b regulate DNA replication licensing, tumorigenesis, and prognosis by targeting CDC6 in lung cancer. Mol Cancer Res 12: 1535-1546, 2014.

9. Zhang Z, Kim K, Li X, Moreno M, Sharp T, Goodheart MJ, Safe S, Dupuy AJ and Amendt BA: MicroRNA-26b represses colon cancer cell proliferation by inhibiting lymphoid enhancer factor 1 expression. Mol Cancer Ther 13: 1942-1951, 2014.

10. Lin C, Wang Z, Li L, He Y, Fan J, Liu Z, Zhao S and Ju D: The role of autophagy in the cytotoxicity induced by recombinant human arginase in laryngeal squamous cell carcinoma. Appl Microbiol Biotechnol 99: 8487-8494, 2015.

11. Pereira DL, Dos Santos Ferreira AC, de Faria GP and Kwee JK: Autophagy interplays with apoptosis and cell cycle regulation in the growth inhibiting effect of Trisenox in HEP-2, a laryngeal squamous cancer. Pathol Oncol Res 21: 103-111, 2015.

12. Li R, Tan S, Yu M, Jundt MC, Zhang S and Wu M: Annexin A2 regulates autophagy in Pseudomonas aeruginosa infection through the Akt1-mTOR-ULK1/2 signaling pathway. J Immunol 195: 3901-3911,2015.

13. Miki Y, Tanji K, Mori F, Utsumi J, Sasaki H, Kakita A, Takahashi $\mathrm{H}$ and Wakabayashi $\mathrm{K}$ : Alteration of upstream autophagy-related proteins (ULK1, ULK2, Beclin1, VPS34 and AMBRA1) in Lewy body disease. Brain Pathol 26: 359-370, 2016.

14. Shukla S, Patric IR, Patil V, Shwetha SD, Hegde AS, Chandramouli BA, Arivazhagan A, Santosh V and Somasundaram K: Methylation silencing of ULK2, an autophagy gene, is essential for astrocyte transformation and tumor growth. J Biol Chem 289: 22306-22318, 2014

15. Snietura M, Jaworska M, Mlynarczyk-Liszka J, Goraj-Zajac A, Piglowski W, Lange D, Wozniak G, Nowara E and Suwinski R: PTEN as a prognostic and predictive marker in postoperative radiotherapy for squamous cell cancer of the head and neck. PLoS One 7: e33396, 2012.

16. Yang JQ, Liang Z, Wu M, Sun YM and Liu HX: Expression of p27 and PTEN and clinical characteristics in early laryngeal squamous cell carcinoma and their correlation with recurrence. Int J Clin Exp Pathol 8: 5715-5720, 2015.

17. Guo Q and Li Y: Research on the expression of Survivin and PTEN in laryngeal squamous cell carcinoma transplanted on the back sides of nude mice treated by gold throat tablets. Lin Chung Er Bi Yan Hou Tou Jing Wai Ke Za Zhi 26: 1134-1137, 1143, 2012 (In Chinese).

18. Yang N, Hui L, Wang Y, Yang H and Jiang X: SOX2 promotes the migration and invasion of laryngeal cancer cells by induction of MMP-2 via the PI3K/Akt/mTOR pathway. Oncol Rep 31: 2651-2659, 2014.
19. García-Carracedo D, Villaronga MA, Álvarez-Teijeiro S, Hermida-Prado F, Santamaría I, Allonca E, Suárez-Fernández L, Gonzalez MV, Balbín M, Astudillo A, et al: Impact of $\mathrm{PI} 3 \mathrm{~K} / \mathrm{AKT} / \mathrm{mTOR}$ pathway activation on the prognosis of patient with head and neck squamous cell carcinomas. Oncotarget 7: 29780-29793, 2016.

20. Bao YY, Zhou SH, Lu ZJ, Fan J and Huang YP: Inhibiting GLUT-1 expression and PI3K/Akt signaling using apigenin improves the radiosensitivity of laryngeal carcinoma in vivo. Oncol Rep 34: 1805-1814, 2015.

21. Wang J, Zhao X, Pan X, Zhao L, Zhou J and Ji M: The role of primary surgical treatment in young patients with squamous cell carcinoma of the larynx: A 20-year review of 34 cases. World J Surg Oncol 13: 283, 2015.

22. Zhong JT and Zhou SH: Warburg effect, hexokinase-II, and radioresistance of laryngeal carcinoma. Oncotarget 8: 14133-14146, 2017.

23. Yoo GH, Moon J, Leblanc M, Lonardo F, Urba S, Kim H, Hanna E, Tsue T, Valentino J, Ensley J, et al: A phase 2 trial of surgery with perioperative INGN 201 (Ad5CMV-p53) gene therapy followed by chemoradiotherapy for advanced, resectable squamous cell carcinoma of the oral cavity, oropharynx, hypopharynx, and larynx: Report of the Southwest Oncology Group. Arch Otolaryngol Head Neck Surg 135: 869-874, 2009.

24. Mirisola V, Mora R, Esposito AI, Guastini L, Tabacchiera F, Paleari L, Amaro A, Angelini G, Dellepiane M, Pfeffer U, et al: A prognostic multigene classifier for squamous cell carcinomas of the larynx. Cancer Lett 307: 37-46, 2011.

25. Gao S, Wang J, Xie J, Zhang T and Dong P: Role of miR-138 in the regulation of larynx carcinoma cell metastases. Tumour Biol, 2015.

26. Espinoza-Mellado MR, Reyes-Picaso C, Garcés-Pérez MS, Jardón-Serrano CV, López-Villegas EO and Giono-Cerezo S: Haemophilus influenzae triggers autophagy in HEp-2 cells. Arch Microbiol 198: 199-204, 2016.

27. Yuan P, Cao W, Zang Q, Li G, Guo X and Fan J: The HIF-2 $\alpha$ MALAT1-miR-216b axis regulates multi-drug resistance of hepatocellular carcinoma cells via modulating autophagy. Biochem Biophys Res Commun 478: 1067-1073, 2016.

28. Chan EY, Kir S and Tooze SA: siRNA screening of the kinome identifies ULK1 as a multidomain modulator of autophagy. J Biol Chem 282: 25464-25474, 2007.

29. John Clotaire DZ,Zhang B, Wei N, Gao R, Zhao F, Wang Y, Lei M and Huang W: MiR-26b inhibits autophagy by targeting ULK2 in prostate cancer cells. Biochem Biophys Res Commun 472: 194-200, 2016.

30. Jung CH, Jun CB, Ro SH, Kim YM, Otto NM, Cao J, Kundu M and Kim DH: ULK-Atg13-FIP200 complexes mediate mTOR signaling to the autophagy machinery. Mol Biol Cell 20: 1992-2003, 2009.

31. Phadngam S, Castiglioni A, Ferraresi A, Morani F, Follo C and Isidoro C: PTEN dephosphorylates AKT to prevent the expression of GLUT1 on plasmamembrane and to limit glucose consumption in cancer cells. Oncotarget 7: 84999-85020, 2016.

32. Kan X, Sun Y, Lu J, Li M, Wang Y, Li Q, Liu Y, Liu M and Tian L: Co-inhibition of miRNA-21 and miRNA-221 induces apoptosis by enhancing the p53-mediated expression of pro-apoptotic miRNAs in laryngeal squamous cell carcinoma. Mol Med Rep 13: 4315-4320, 2016.

33. Palumbo T, Faucz FR, Azevedo M, Xekouki P, Iliopoulos D and Stratakis CA: Functional screen analysis reveals miR-26b and miR-128 as central regulators of pituitary somatomammotrophic tumor growth through activation of the PTEN-AKT pathway. Oncogene 32: 1651-1659, 2013.

34. Zhang XJ and Jia SS: Fisetin inhibits laryngeal carcinoma through regulation of AKT/NF- $\mathrm{KB} / \mathrm{mTOR}$ and ERK1/2 signaling pathways. Biomed Pharmacother 83: 1164-1174, 2016. 